\title{
An electromechanical higher order model for piezoelectric functionally graded plates
}

\author{
S. M. Shiyekar • Tarun Kant
}

Received: 19 March 2010/Accepted: 31 March 2010/Published online: 23 April 2010

(C) Springer Science+Business Media, B.V. 2010

\begin{abstract}
Bidirectional flexure analysis of functionally graded (FG) plate integrated with piezoelectric fiber reinforced composites (PFRC) is presented in this paper. A higher order shear and normal deformation theory (HOSNT12) is used to analyze such hybrid or smart FG plate subjected to electromechanical loading. The displacement function of the present model is approximated as Taylor's series in the thickness coordinate, while the electro-static potential is approximated as layer wise linear through the thickness of the PFRC layer. The equations of equilibrium are obtained using principle of minimum potential energy and solution is by Navier's technique. Elastic constants are varying exponentially along thickness ( $z$ axis) for FG material while Poisson's ratio is kept constant. PFRC actuator attached either at top or bottom of FG plate and analyzed under mechanical and coupled mechanical and electrical loading. Comparison of present HOSNT12 is made with exact and finite element method (FEM).
\end{abstract}

Keywords Higher order theory · Piezoelectric fiber reinforced composites - Functionally Graded

\footnotetext{
S. M. Shiyekar ( $₫)$

Department of Civil Engineering, Sinhgad College of Engineering, Vadgaon (Bk), Pune 411 041, India e-mail: smshiyekar.scoe@sinhgad.edu

T. Kant

Department of Civil Engineering, Indian Institute of Technology Bombay, Powai 400 076, India e-mail: tkant@civil.iitb.ac.in
}

\section{Introduction}

Piezoelectric materials transform elastic field into the electric field and converse behavior leads many researchers to study their controlling capabilities applicable to structures like plates and shells. Such structures are called as smart, intelligent, adaptive as well as hybrid structures. In conventional composites failure occurs at interface due to abrupt change in material properties. Elastic properties are varying smoothly across the thickness of the FG material and hence failure due to de lamination is avoided.

Piezoelectric materials show coupling phenomenon between elastic and electric fields. Tiersten and Mindlin (1962) initiated work on piezoelectric plates. Further Tiersten (1969) contributed this work by exploring the governing equations of linear piezoelectric continuum by analyzing vibrations of a single piezoelectric layer.

Monolithic piezoelectric materials exhibit very low stress/strain coefficients and hence low controlling capabilities. Smith and Auld (1991) presented micromechanical analysis of vertically reinforced piezoelectric composites with slight increase in the stress/ strain piezoelectric coefficients.

Mallik and Ray (2003) proposed the concept of unidirectional piezoelectric fiber reinforced composite (PFRC) materials and presented their effective elastic and piezoelectric properties. Piezoelectric stress/strain coefficients are improved considerably as compared to monolithic piezoelectric materials. Vertically 
reinforced piezoelectric composites are not suitable for the bending mode actuation (Mallik and Ray 2003).

Many investigators studied hybrid composite laminates using various plate theories. Kapuria and Dumir (2000) presented coupled first-order shear deformation theory for hybrid laminated plates subjected to thermoelectrical loading. Elasticity solutions always serve as benchmark for other approximate solutions. Ray et al. (1993) developed three-dimensional (3D) elasticity solutions for intelligent plate simply supported and perfectly bonded with distributed Polyvinylidene Fluoride (PVDF) piezoelectric layers at top and bottom and presented static displacement control for different span to depth ratios. Heyliger (1994) obtained exact solution for an unsymmetric cross ply composite laminate attached with PZT-4 layers of piezoelectric material at upper and lower surfaces. The 3D solution methodology used by Ray and Heyliger is the same as the work of Pagano (1970) for laminated composite plates. Later Heyliger (1997) provided the 3D exact solution for single and two layers of piezoelectric materials. Vel and Batra (2000) used Eshelby-Stroh formulation to obtained 3D elasticity solution to analyze multilayered piezoelectric plate for arbitrary boundary conditions. Batra and Vel (2001) presented exact thermo elastic solutions for FG plates, while Sankar (2001) presented 3D exact solutions for functionally graded beams under mechanical pressure. Ray and Sachade (2006a, b) reported 3D elasticity solution and FEM for FG plate attached with PFRC actuator. Reddy and Cheng (2001) also presented elasticity solutions for smart FG plate.

Higher order shear deformation theories (HOST) incorporate transverse shear and normal deformation by expanding the primary displacement fields. Initially Kant (1982) developed complete set of variationally consistent governing equations of equilibrium and presented first FE model based on HOST (Kant et al. 1982). Pandya and Kant $(1987,1988)$ and Kant and Manjunatha $(1988,1994)$ extended the HOST for unsymmetric laminates. Further Kant and Swaminathan (2002) presented a refined higher order theory and discussed analytical solution for sandwiches and laminates. Reddy (1984) presented a simple third order theory for laminates maintaining zero shear stress conditions at boundaries of the thickness dimension.

In this paper, a higher order shear and normal deformation theory (HOSNT12) is used to model the elastic displacements of FG plate whereas; electric potential in the PFRC actuator layer is modeled as piece wise linear.

\section{Formulation}

Consider bidirectional flexure of functionally graded (FG) plate. At $x=0, a$ and at $y=0, b$ the FG plate is simply supported and attached with distributed PFRC actuator of thickness $t_{p}$ at top or bottom as shown in the Fig. 1. Span of the hybrid FG plate is $a$ along $x$ axis and $b$ along $y$ axis in Cartesian coordinate system. Thickness of FG plate is $h$ along $z$ axis and located at $-h / 2$ and $+h / 2$ from bottom and top of FG plate, respectively. Electrostatic potential is applied at top of PFRC actuator $\left(h / 2+t_{p}\right)$ and FG plate is electrically grounded at $z=+h / 2$.

Displacement components $u(x, y, z), v(x, y, z)$ and $w(x, y, z)$ at any point in the plate are expanded in a
Fig. 1 Geometry of Functionally Graded (FG) simply (diaphragm) supported along all edges plate attached with PFRC actuator

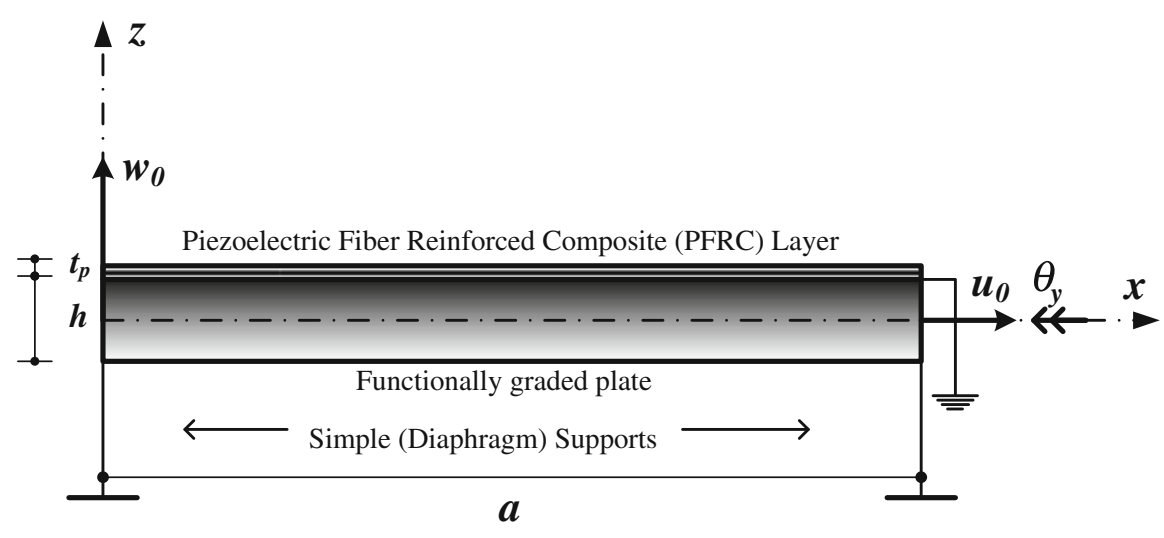


Taylor's series to approximate the three-dimensional (3D) elasticity problem as a two-dimensional (2D) plate problem. The assumed displacement field of present higher order shear and normal deformation model is in the following form:

Model HOSNT12: (Kant and Swaminathan 2002)

$$
\begin{aligned}
& u(x, y, z)=u_{0}(x, y)+z \theta_{x}(x, y)+z^{2} u_{0}^{*}(x, y)+z^{3} \theta_{x}^{*}(x, y) \\
& v(x, y, z)=v_{0}(x, y)+z \theta_{y}(x, y)+z^{2} v_{0}^{*}(x, y)+z^{3} \theta_{y}^{*}(x, y) \\
& w(x, y, z)=w_{0}(x, y)+z \theta_{z}(x, y)+z^{2} w_{0}^{*}(x, y)+z^{3} \theta_{z}^{*}(x, y)
\end{aligned}
$$

where the parameters $u_{0}, v_{0}$ are in-plane displacements and $w_{0}$ is transverse displacement at any point $(x, y)$ on the middle plane of the plate. $\theta_{x} \& \theta_{y}$ are the rotations of the normal to the mid-plane about $y$ and $x$ axes, respectively. Other parameters such as $u_{0}^{*}, \theta_{x}^{*}, v_{0}^{*}, \theta_{y}^{*}$, $w_{0}^{*}, \theta_{z}^{*}$ are the corresponding higher order terms in the Taylor series expansion at mid-plane.

Strain displacement relationship as per classical theory of elasticity is written in Eq. 2 as

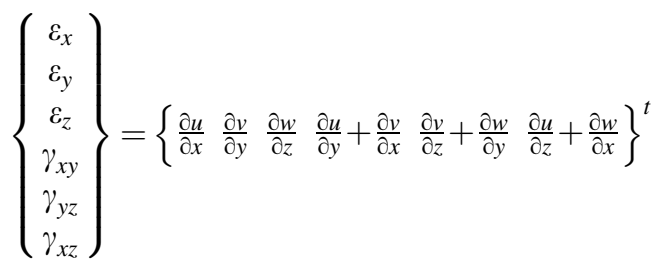

Tiersten (1969) presented linear constitutive equation which couples the elastic and electric field for a single piezoelectric layer as

$$
\begin{aligned}
\{\sigma\} & =[C]\{\varepsilon\}-[e]\{E\}^{L} \\
\{D\} & =[e]^{t}\{\varepsilon\}+[\eta]\{E\}^{L}
\end{aligned}
$$

The electric field intensity vector $E$ related to electrostatic potential $\xi(x, y, z)$ in the $L$ th layer is given by

$$
\begin{aligned}
E_{x}^{L} & =-\frac{\partial \xi(x, y, z)^{L}}{\partial x}, \quad E_{y}^{L}=-\frac{\partial \xi(x, y, z)^{L}}{\partial y}, \\
E_{z}^{L} & =-\frac{\partial \xi(x, y, z)^{L}}{\partial z}
\end{aligned}
$$

where $\sigma, Q, \varepsilon, e, E, D$ and $\eta$ are, stress vector, elastic constant matrix, strain vector, piezoelectric constant matrix, electric field intensity vector, electric displacement vector and dielectric constant matrix, respectively. Effective piezoelectric constant matrix $e$ and dielectric matrix $\eta$ for PFRC layer aligned at zero angle with respect to $x$-axis is given by Mallik and Ray (2003) as

$$
[e]=\left[\begin{array}{ccc}
0 & 0 & e_{31} \\
0 & 0 & e_{32} \\
0 & 0 & e_{33} \\
0 & 0 & 0 \\
0 & e_{24} & 0 \\
e_{15} & 0 & 0
\end{array}\right], \quad[\eta]=\left[\begin{array}{ccc}
\eta_{11} & 0 & 0 \\
0 & \eta_{22} & 0 \\
0 & 0 & \eta_{33}
\end{array}\right]
$$

The first set of Eq. 3 is divided in two components of stresses. One is elastic stress component (es) and other is piezoelectric stress component $(p z)$.

First set of Eq. 3 is written as $\{\sigma\}=\{\sigma\}^{e s}-\{\sigma\}^{p z}$ where

$$
\begin{aligned}
& \{\sigma\}^{e s}=\left[\begin{array}{cccccc}
C_{11} & C_{12} & C_{13} & 0 & 0 & 0 \\
C_{12} & C_{22} & C_{23} & 0 & 0 & 0 \\
C_{13} & C_{23} & C_{33} & 0 & 0 & 0 \\
0 & 0 & 0 & C_{44} & 0 & 0 \\
0 & 0 & 0 & 0 & C_{55} & 0 \\
0 & 0 & 0 & 0 & 0 & C_{66}
\end{array}\right]^{L}\left\{\begin{array}{c}
\varepsilon_{x} \\
\varepsilon_{y} \\
\varepsilon_{z} \\
\gamma_{x y} \\
\gamma_{y z} \\
\gamma_{x z}
\end{array}\right\}, \\
& \{\sigma\}^{p z}=\left[\begin{array}{ccc}
0 & 0 & e_{31} \\
0 & 0 & e_{32} \\
0 & 0 & e_{33} \\
0 & 0 & 0 \\
0 & e_{24} & 0 \\
e_{15} & 0 & 0
\end{array}\right]^{L}\left\{\begin{array}{l}
-\frac{\partial \xi(x, y, z)}{\partial x} \\
-\frac{\partial \xi(x, y, z)}{\partial y} \\
-\frac{\partial \xi(x, y, z)}{\partial z}
\end{array}\right\}
\end{aligned}
$$

and $C_{i j}$ are elastic constants of FG plate and defined as per isotropic layer.

$$
\begin{gathered}
C_{11}=C_{22}=C_{33}=\frac{(1-v) E(z)}{(1+v)(1-2 v)} ; \\
C_{12}=C_{13}=C_{23}=\frac{v E(z)}{(1+v)(1-2 v)} ; \\
C_{44}=C_{55}=C_{66}=\frac{E(z)}{2(1+v)}=G .
\end{gathered}
$$

Young's modulus of FG material is a function of $z$ coordinate and governed by exponential law (Sankar 2001), where as Poisson's ratio is kept constant.

$E(z)=E_{0} e^{\lambda(z+h / 2)}$

where $E_{0}$ is Young's modulus of FG material at bottom and $\lambda$ is a non-homogeneous parameter of the FG material across the thickness. 
Stress resultants are also defined as elastic and piezoelectric stress resultants.

Elastic stress resultants $[Q, S, M, N]^{e s}$

$$
\begin{aligned}
& {\left[Q_{x}^{e s}, Q_{y}^{e s} \mid Q_{x}^{e s^{*}}, Q_{y}^{e s^{*}}\right]} \\
& =\sum_{L=1}^{n} \int_{-h / 2}^{+h / 2}\left\{\tau_{x z}^{e s}, \tau_{y z}^{e s}\right\}\left[1 \mid z^{2}\right] d z,\left[S_{x}^{e s}, S_{y}^{e s} \mid S_{x}^{e s^{*}}, S_{y}^{e s^{*}}\right] \\
& \quad=\sum_{L=1}^{n} \int_{-h / 2}^{+h / 2}\left\{\tau_{x z}^{e s}, \tau_{y z}^{e s}\right\}\left[z \mid z^{3}\right] d z \\
& {\left[M_{x}^{e s}, M_{y}^{e s}, M_{x y}^{e s} \mid M_{x}^{e s^{*}}, M_{y}^{e s^{*}}, M_{x y}^{e s^{*}}\right]} \\
& \quad=\sum_{L=1}^{n} \int_{-h / 2}^{+h / 2}\left\{\sigma_{x}^{e s}, \sigma_{y}^{e s}, \tau_{x y}^{e s}\right\}\left[z \mid z^{3}\right] d z, M_{z}^{e s} \\
& \quad=\sum_{L=1}^{n} \int_{-h / 2}^{+h / 2} \sigma_{z}^{e} d z, \\
& {\left[N_{x}^{e s}, N_{y}^{e s}, N_{z}^{e s}, N_{x y}^{e s} \mid N_{x}^{e s^{*}}, N_{y}^{e s^{*}}, N_{z}^{e s^{*}}, N_{x y}^{e s^{*}}\right]} \\
& \quad=\sum_{L=1}^{n} \int_{-h / 2}^{+h / 2}\left\{\sigma_{x}^{e s}, \sigma_{y}^{e s}, \sigma_{z}^{e s}, \tau_{x y}^{e s}\right\}\left[1 \mid z^{2}\right] d z .
\end{aligned}
$$

Piezoelectric stress resultants $[Q, S, M, N]^{p z}$

$$
\begin{aligned}
& {\left[Q_{x}^{p z}, Q_{y}^{p z} \mid Q_{x}^{p z^{*}}, Q_{y}^{p z^{*}}\right]} \\
& =\int_{+h / 2}^{h / 2+t p}\left\{\tau_{x z}^{p z}, \tau_{y z}^{p z}\right\}\left[1 \mid z^{2}\right] d z,\left[S_{x}^{p z}, S_{y}^{p z} \mid S_{x}^{p z^{*}}, S_{y}^{p z^{*}}\right] \\
& =\int_{+h / 2}^{h / 2+t p}\left\{\tau_{x z}^{p z}, \tau_{y z}^{p z}\right\}\left[z \mid z^{3}\right] d z \\
& {\left[M_{x}^{p z}, M_{y}^{p z}, M_{x y}^{p z} \mid M_{x}^{p z^{*}}, M_{y}^{p z^{*}}, M_{x y}^{p z^{*}}\right]} \\
& =\int_{+h / 2}^{h / 2+t p}\left\{\sigma_{x}^{p z}, \sigma_{y}^{p z}, \tau_{x y}^{p z}\right\}\left[z \mid z^{3}\right] d z, M_{z}^{p z}=\int_{+h / 2}^{h / 2+t p} \sigma_{z}^{p z} d z, \\
& {\left[N_{x}^{p z}, N_{y}^{p z}, N_{z}^{p z}, N_{x y}^{p z} \mid N_{x}^{p z^{*}}, N_{y}^{p z^{*}}, N_{z}^{p z^{*}}, N_{x y}^{p z^{*}}\right]} \\
& =\int_{+h / 2}^{h / 2+t p}\left\{\sigma_{x}^{p z}, \sigma_{y}^{p z}, \sigma_{z}^{p z}, \tau_{x y}^{p z}\right\}\left[1 \mid z^{2}\right] d z .
\end{aligned}
$$

Total stress resultants $(p z)$ : Total stress resultants $[Q, S, M, N]$ are algebraic sum of elastic and piezoelectric stress resultants.

$$
[Q, S, M, N]=[Q, S, M, N]^{e s}+[Q, S, M, N]^{p z}
$$

Governing equations of equilibrium.

Using principal of minimum potential energy, the equations of equilibrium are obtained as

$$
\begin{array}{cl}
\delta u_{0}: \frac{\partial N_{x}}{\partial x}+\frac{\partial N_{x y}}{\partial y}=0 & \delta u_{0}^{*}: \frac{\partial N_{x}^{*}}{\partial x}+\frac{\partial N_{x y}^{*}}{\partial y}-2 S_{x}=0 \\
\delta v_{0}: \frac{\partial N_{y}}{\partial y}+\frac{\partial N_{x y}}{\partial x}=0 & \delta v_{0}^{*}: \frac{\partial N_{y}^{*}}{\partial y}+\frac{\partial N_{x y}^{*}}{\partial x}-2 S_{y}=0 \\
\delta w_{0}: \frac{\partial Q_{x}}{\partial x}+\frac{\partial Q_{y}}{\partial y}+\left(q_{z}^{+}\right)=0 & \delta w_{0}^{*}: \frac{\partial Q_{x}^{*}}{\partial x}+\frac{\partial Q_{y}^{*}}{\partial y}-2 M_{z}^{*}+\frac{h^{2}}{4}\left(q_{z}^{+}\right)=0 \\
\delta \theta_{x}: \frac{\partial M_{x}}{\partial x}+\frac{\partial M_{x y}}{\partial y}-Q_{x}=0 & \delta \theta_{x}^{*}: \frac{\partial M_{x}^{*}}{\partial x}+\frac{\partial M_{x y}^{*}}{\partial y}-3 Q_{x}^{*}=0 \\
\delta \theta_{y}: \frac{\partial M_{y}}{\partial y}+\frac{\partial M_{x y}}{\partial x}-Q_{y}=0 & \delta \theta_{y}^{*}: \frac{\partial M_{y}^{*}}{\partial y}+\frac{\partial M_{x y}^{*}}{\partial x}-3 Q_{y}^{*}=0 \\
\delta \theta_{z}: \frac{\partial S_{x}}{\partial x}+\frac{\partial S_{y}}{\partial y}-N_{z}+\frac{h}{2}\left(q_{z}^{+}\right)=0 & \delta \theta_{z}^{*}: \frac{\partial S_{x}}{\partial x}+\frac{\partial S_{y}}{\partial y}-3 N_{z}^{*}+\frac{h^{3}}{8}\left(q_{z}^{+}\right)=0
\end{array}
$$


Total stress resultants are the addition of elastic and piezoelectric stress resultants.

Following are the mechanical boundary conditions used for simply supported plate

At edges $x=0$ and $x=a$ :

$v_{0}=0, w_{0}=0, \theta_{y}=0, \theta_{z}=0$,

$$
\begin{aligned}
& M_{x}=0, N_{x}=0, v_{0}^{*}=0, w_{0}^{*}=0, \\
& \theta_{y}^{*}=0, \theta_{z}^{*}=0, M_{x}^{*}=0, N_{x}^{*}=0 .
\end{aligned}
$$

At edges $y=0$ and $y=b$ :

$$
\begin{gathered}
u_{0}=0, w_{0}=0, \theta_{x}=0, \theta_{z}=0, \\
M_{y}=0, N_{y}=0, u_{0}^{*}=0, w_{0}^{*}=0, \\
\theta_{x}^{*}=0, \theta_{z}^{*}=0, M_{y}^{*}=0, N_{y}^{*}=0 .
\end{gathered}
$$

Navier's solution procedure is adopted to find the solution of displacement variables, satisfying the above boundary conditions and is expressed as follows: layer is assumed to be linear through the thickness of the PFRC layer.

$$
\xi_{m n}(z)=\left(\frac{V_{m n}^{t}}{t_{p}}\right) z-\left(\frac{V_{m n}^{t} h}{2 t_{p}}\right)
$$

Equation 15 gives linear variation of thought thickness electro-static potential in the PFRC actuating layer. $V_{m n}^{t}$ represents amplitude of doubly sinusoidal electrostatic potential applied at top of the PFRC whereas; $h$ and $t_{p}$ are thicknesses of elastic FG plate and PFRC layer, respectively. Assumed electrostatic potential satisfies zero electric potential at interface. Similar electrostatic potential can be assumed for PFRC layer at bottom of FG plate.

The piezoelectric stress vectors are calculated from second set of Eq. 6 by substituting actuating electric function (Eq. 15) when either top or bottom voltages $\left(V_{m n}^{t}, V_{m n}^{b}\right)$ are applied. Finally, piezoelectric

$$
\begin{array}{rlrl}
u_{0} & =\sum_{m=1}^{\infty} \sum_{n=1}^{\infty} u_{0_{m n}} \cos \left(\frac{m \pi x}{a}\right) \sin \left(\frac{n \pi y}{b}\right) & u_{0}^{*}=\sum_{m=1}^{\infty} \sum_{n=1}^{\infty} u_{0_{m n}}^{*} \cos \left(\frac{m \pi x}{a}\right) \sin \left(\frac{n \pi y}{b}\right) \\
v_{0}=\sum_{m=1}^{\infty} \sum_{n=1}^{\infty} v_{0_{m n}} \sin \left(\frac{m \pi x}{a}\right) \cos \left(\frac{n \pi y}{b}\right) & v_{0}^{*}=\sum_{m=1}^{\infty} \sum_{n=1}^{\infty} v_{0_{m n}}^{*} \sin \left(\frac{m \pi x}{a}\right) \cos \left(\frac{n \pi y}{b}\right) \\
w_{0}=\sum_{m=1}^{\infty} \sum_{n=1}^{\infty} w_{0_{m n}} \sin \left(\frac{m \pi x}{a}\right) \sin \left(\frac{n \pi y}{b}\right) & w_{0}^{*}=\sum_{m=1}^{\infty} \sum_{n=1}^{\infty} w_{0_{m n}}^{*} \sin \left(\frac{m \pi x}{a}\right) \sin \left(\frac{n \pi y}{b}\right) \\
\theta_{x}=\sum_{m=1}^{\infty} \sum_{n=1}^{\infty} \theta_{x_{m n}} \cos \left(\frac{m \pi x}{a}\right) \sin \left(\frac{n \pi y}{b}\right) & \theta_{x}^{*}=\sum_{m=1}^{\infty} \sum_{n=1}^{\infty} \theta_{x m n}{ }^{*} \cos \left(\frac{m \pi x}{a}\right) \sin \left(\frac{n \pi y}{b}\right) \\
\theta_{y}=\sum_{m=1}^{\infty} \sum_{n=1}^{\infty} \theta_{y_{m n}} \sin \left(\frac{m \pi x}{a}\right) \cos \left(\frac{n \pi y}{b}\right) & \theta_{y}^{*}=\sum_{m=1}^{\infty} \sum_{n=1}^{\infty} \theta_{y_{m n}} \sin \left(\frac{m \pi x}{a}\right) \cos \left(\frac{n \pi y}{b}\right) \\
\theta_{z}=\sum_{m=1}^{\infty} \sum_{n=1}^{\infty} \theta_{z_{m n}} \sin \left(\frac{m \pi x}{a}\right) \sin \left(\frac{n \pi y}{b}\right) & \theta_{z}^{*}=\sum_{m=1}^{\infty} \sum_{n=1}^{\infty} \theta_{z_{m n}} \sin \left(\frac{m \pi x}{a}\right) \sin \left(\frac{n \pi y}{b}\right) \\
q_{z}^{+}=\sum_{m=1}^{\infty} \sum_{n=1}^{\infty} q_{z_{m n}}^{+} \sin \left(\frac{m \pi x}{a}\right) \sin \left(\frac{n \pi y}{b}\right) & \xi(x, z)=\sum_{m=1}^{\infty} \sum_{n=1}^{\infty} \xi_{m n}(z) \sin \left(\frac{m \pi x}{a}\right) \sin \left(\frac{n \pi y}{b}\right)
\end{array}
$$

where $q_{z}^{+}$is the mechanical loading term and $\xi(x, z)$ is the electrical loading term. Through thickness electric potential $\xi_{m n}(z)$ is assumed as per the following.

The elastic FG layer is attached with distributed actuator layer of PFRC. The thickness of the PFRC layer is small as compared to the thickness of the substrate, the electro-static potential in the actuator stress resultants are evaluated from Eq. 10. Similarly elastic stress vectors and elastic stress resultants are calculated from first set of Eqs. 6 and 9, respectively. Displacement terms are obtained by solving linear algebraic equations by substituting total stress resultants from Eq. 11 in a set of equilibrium equations (Eq. 12). 


\section{Numerical results and discussions}

Numerical evaluation of a FG plate attached with piezoelectric layer either at top or at bottom is considered. Property of piezoelectric fiber reinforced composite is taken as follows (Ray and Sachade 2006b).

$F G$ Material: $E_{0}=200 \mathrm{GPa}$ and $v=0.3$.

PFRC (PZT5H): $\mathrm{C}_{11}=32.6 \mathrm{GPa}, \mathrm{C}_{11}=4.3 \mathrm{GPa}$, $\mathrm{C}_{22}=7.2 \mathrm{GPa}, \mathrm{C}_{44}=1.05 \mathrm{GPa}, \mathrm{C}_{55}=\mathrm{C}_{44}=1.29$ $\mathrm{GPa}, \mathrm{e}_{31}=-6.76 \mathrm{C} / \mathrm{m}^{2}, \mathrm{e}_{32}=\mathrm{e}_{15}=\mathrm{e}_{24}=\mathrm{e}_{33}=0.000$, $\varepsilon_{11}=\varepsilon_{22}=0.037 \times 10^{-9} \mathrm{~F} / \mathrm{m}^{2}, \varepsilon_{33}=10.64 \times 10^{-9} \mathrm{~F} / \mathrm{m}^{2}$.

Results for displacements and stresses are obtained for following cases.
Case I: $\mathrm{E}_{\mathrm{h}} / \mathrm{E}_{0}=10, q_{z_{m n}}^{+}=-40 \mathrm{~N} / \mathrm{m}^{2}, V_{m n}^{t}=0$, $100,-100, \mathrm{~S}=10,20,100, \mathrm{PFRC}$ at top

Case II: $\mathrm{E}_{\mathrm{h}} / \mathrm{E}_{0}=0.1, q_{z_{m n}}^{+}=-40 \mathrm{~N} / \mathrm{m}^{2}, V_{m n}^{t}=0$, $100,-100, \mathrm{~S}=10,20,100, \mathrm{PFRC}$ at top

Case III: $\mathrm{E}_{\mathrm{h}} / \mathrm{E}_{0}=10, q_{z_{m n}}^{+}=-40 \mathrm{~N} / \mathrm{m}^{2}, V_{m n}^{b}=0$, $100,-100, \mathrm{~S}=10,20,100, \mathrm{PFRC}$ at bottom

Case IV: $\mathrm{E}_{\mathrm{h}} / \mathrm{E}_{0}=0.1, q_{z_{m n}}^{+}=-40 \mathrm{~N} / \mathrm{m}^{2}, V_{m n}^{b}=0$, $100,-100, \mathrm{~S}=10,20,100$, PFRC at bottom. Where $E_{h}$ is Young's modulus at top of FG plate.

Results are compared with 3D exact solution (Ray and Sachade 2006a) and FE solutions (Ray and Sachade 2006b). In-plane displacement, transverse

Table 1 FG plates $\left(\mathbf{E}_{\mathrm{h}} / \mathbf{E}_{0}=0.1\right)$ with applied sinusoidal mechanical and electrical loadings I PFRC at top

\begin{tabular}{|c|c|c|c|c|c|c|c|c|c|c|}
\hline \multicolumn{2}{|c|}{ Aspect ratio } & \multicolumn{3}{|l|}{$S=10$} & \multicolumn{3}{|l|}{$\mathrm{S}=20$} & \multicolumn{3}{|l|}{$S=100$} \\
\hline Quantity & Source & $V=0$ & $V=100$ & $V=-100$ & $V=0$ & $V=100$ & $V=-100$ & $V=0$ & $V=100$ & $V=-100$ \\
\hline \multirow[t]{3}{*}{$\bar{w}$} & Present $^{\mathrm{a}}$ & -8.6140 & 3164.12 & -3181.35 & -8.3577 & 790.3310 & -807.046 & -8.2750 & 23.7481 & -40.2981 \\
\hline & $3 D^{b}$ & -8.7397 & 3253.30 & -3270.80 & -8.5437 & 813.7105 & -830.7979 & -8.4806 & 23.9160 & -40.5745 \\
\hline & $\mathrm{FEM}^{\mathrm{c}}$ & -8.6673 & 3212.90 & -3230.30 & -8.4154 & 797.9455 & -814.7764 & -8.3292 & 24.4974 & -41.4587 \\
\hline \multirow[t]{6}{*}{$\bar{u}$} & Present $^{\mathrm{a}}$ & -0.0905 & -3.8486 & 3.6674 & -0.0899 & -1.14816 & 0.988288 & -0.089722 & -0.1336 & -0.04584 \\
\hline & & 0.1653 & -112.9800 & 113.311 & 0.1689 & -26.5921 & 26.9299 & 0.170091 & -0.8807 & 1.22091 \\
\hline & $3 D^{b}$ & -0.0914 & -3.1845 & 3.0017 & -0.0912 & -0.9839 & 0.8015 & -0.0912 & -0.1285 & -0.0539 \\
\hline & & 0.1686 & -114.0117 & 114.3489 & 0.1735 & -27.0971 & 27.4441 & 0.175 & -0.8991 & 1.2493 \\
\hline & $\mathrm{FEM}^{\mathrm{c}}$ & -0.0901 & -3.0418 & 2.8616 & -0.0902 & -1.0641 & 0.8838 & -0.0901 & -0.1322 & -0.0480 \\
\hline & & 0.1714 & -107.7880 & 108.1308 & 0.1716 & -26.3653 & 26.7085 & 0.1715 & -0.8840 & 1.2270 \\
\hline \multirow[t]{6}{*}{$\bar{\sigma}_{x}$} & Present $^{\mathrm{a}}$ & 0.3908 & -33.732 & 34.5138 & 0.3848 & -7.74463 & 8.57438 & 0.38294 & 0.06279 & 0.703096 \\
\hline & & -0.0848 & 47.3070 & -47.4767 & -0.0846 & 11.26 & -11.4293 & -0.0845 & 0.3627 & -0.53194 \\
\hline & $3 D^{b}$ & 0.4042 & -41.0915 & 41.8999 & 0.4031 & -9.8557 & 10.6619 & 0.4027 & -0.0061 & 0.8115 \\
\hline & & -0.0807 & 44.3075 & -44.4689 & -0.0796 & 10.6491 & -10.8083 & -0.0793 & 0.3451 & -0.5036 \\
\hline & FEM $^{\mathrm{c}}$ & 0.4066 & -42.1225 & 42.9357 & 0.4068 & -9.6579 & 10.4715 & 0.4066 & 0.0199 & 0.8013 \\
\hline & & -0.0793 & 43.0275 & -43.1862 & -0.0794 & 10.5905 & -10.7493 & -0.0793 & 0.3460 & -0.5046 \\
\hline \multirow[t]{6}{*}{$\bar{\sigma}_{y}$} & Present $^{\mathrm{a}}$ & 0.3763 & -163.0770 & 163.83 & 0.3697 & -41.0169 & 41.7565 & 0.36762 & -1.29497 & 2.03021 \\
\hline & & -0.0866 & 30.8163 & -30.9899 & -0.0862 & 7.70831 & -7.88081 & -0.08611 & 0.22666 & -0.398894 \\
\hline & $3 \mathrm{D}^{\mathrm{b}}$ & 0.3900 & -170.3199 & 171.0999 & 0.3883 & -43.1562 & 43.9327 & 0.3877 & -1.3654 & 2.1408 \\
\hline & & -0.0825 & 28.1032 & -28.2681 & -0.0812 & 7.1105 & -7.2729 & -0.0808 & 0.2088 & -0.3704 \\
\hline & $\mathrm{FEM}^{\mathrm{c}}$ & 0.3916 & -172.9020 & 173.6851 & 0.3913 & -43.5671 & 44.3496 & 0.3909 & -1.3748 & 2.1565 \\
\hline & & -0.0810 & 28.0609 & -28.2229 & -0.0810 & 7.0834 & -7.2453 & -0.0809 & 0.2072 & -0.3689 \\
\hline \multirow[t]{6}{*}{$\bar{\tau}_{x y}$} & Present $^{\mathrm{a}}$ & -0.2116 & 55.3721 & -55.7955 & -0.20979 & 13.8615 & -14.2811 & -0.2091 & 0.356017 & -0.77434 \\
\hline & & 0.0408 & -19.0577 & 19.1394 & 0.04162 & -4.6504 & 4.7336 & 0.0418 & -0.144775 & 0.228522 \\
\hline & $3 D^{b}$ & -0.2138 & 56.9184 & -57.3461 & -0.2131 & 14.2724 & -14.6986 & -0.2128 & 0.3692 & -0.7948 \\
\hline & & 0.0416 & -19.4500 & 19.5333 & 0.0427 & -4.7790 & 4.8645 & 0.0431 & -0.1491 & 0.2353 \\
\hline & FEM $^{\mathrm{c}}$ & -0.2148 & 57.9332 & -58.3627 & -0.2148 & 14.3377 & -14.7673 & -0.2147 & 0.3667 & -0.7962 \\
\hline & & 0.0431 & -19.1536 & 19.2399 & 0.0431 & -4.7614 & 4.8477 & 0.0431 & -0.1489 & 0.2352 \\
\hline
\end{tabular}

\footnotetext{
${ }^{a}$ Present-HOSNT12 using linear variation of electrostatic potential through the thickness

b 3D-Exact (Ray and Sachade 2006a)

${ }^{c}$ FEM-FOST based (Ray and Sachade 2006b)
} 


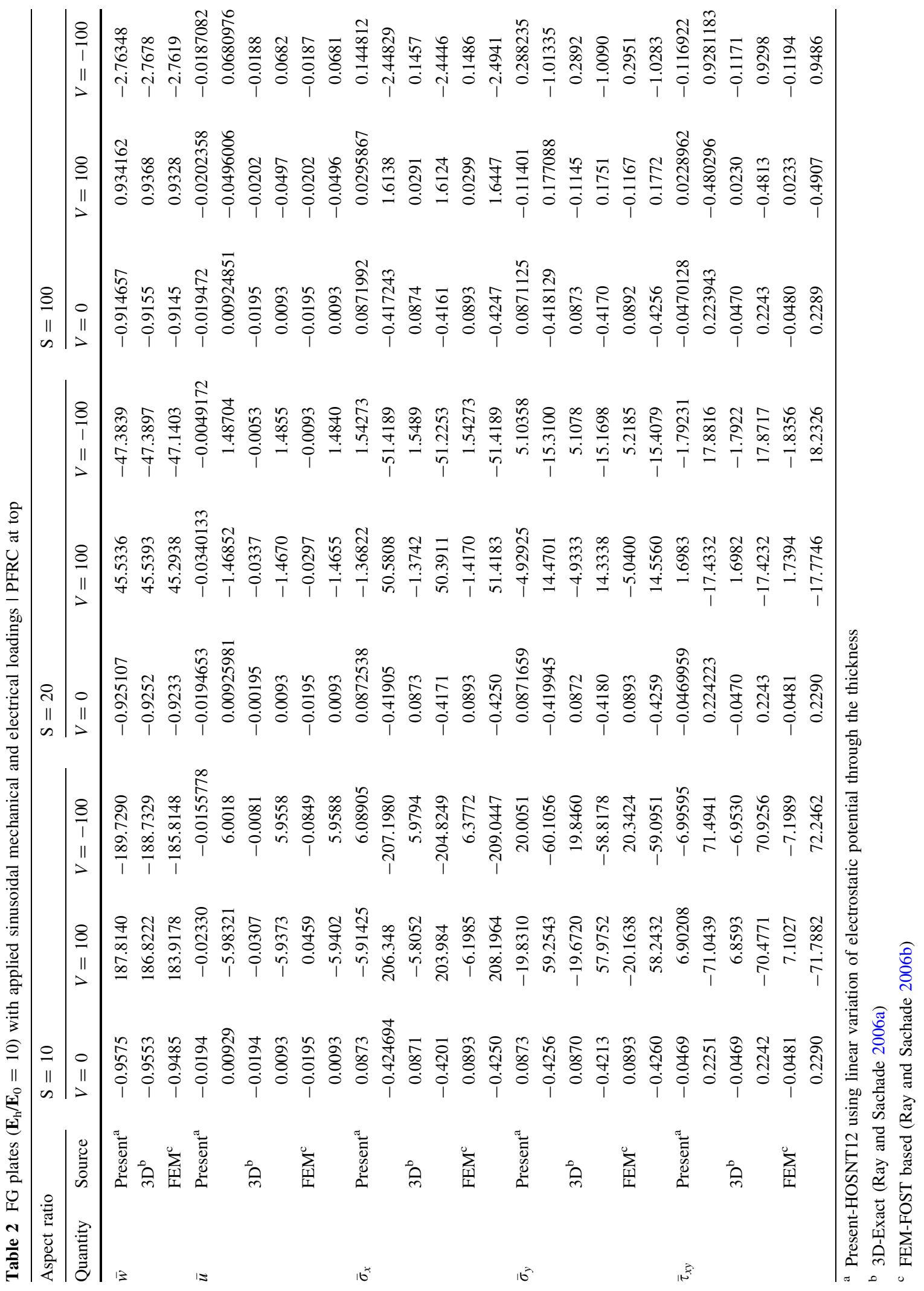




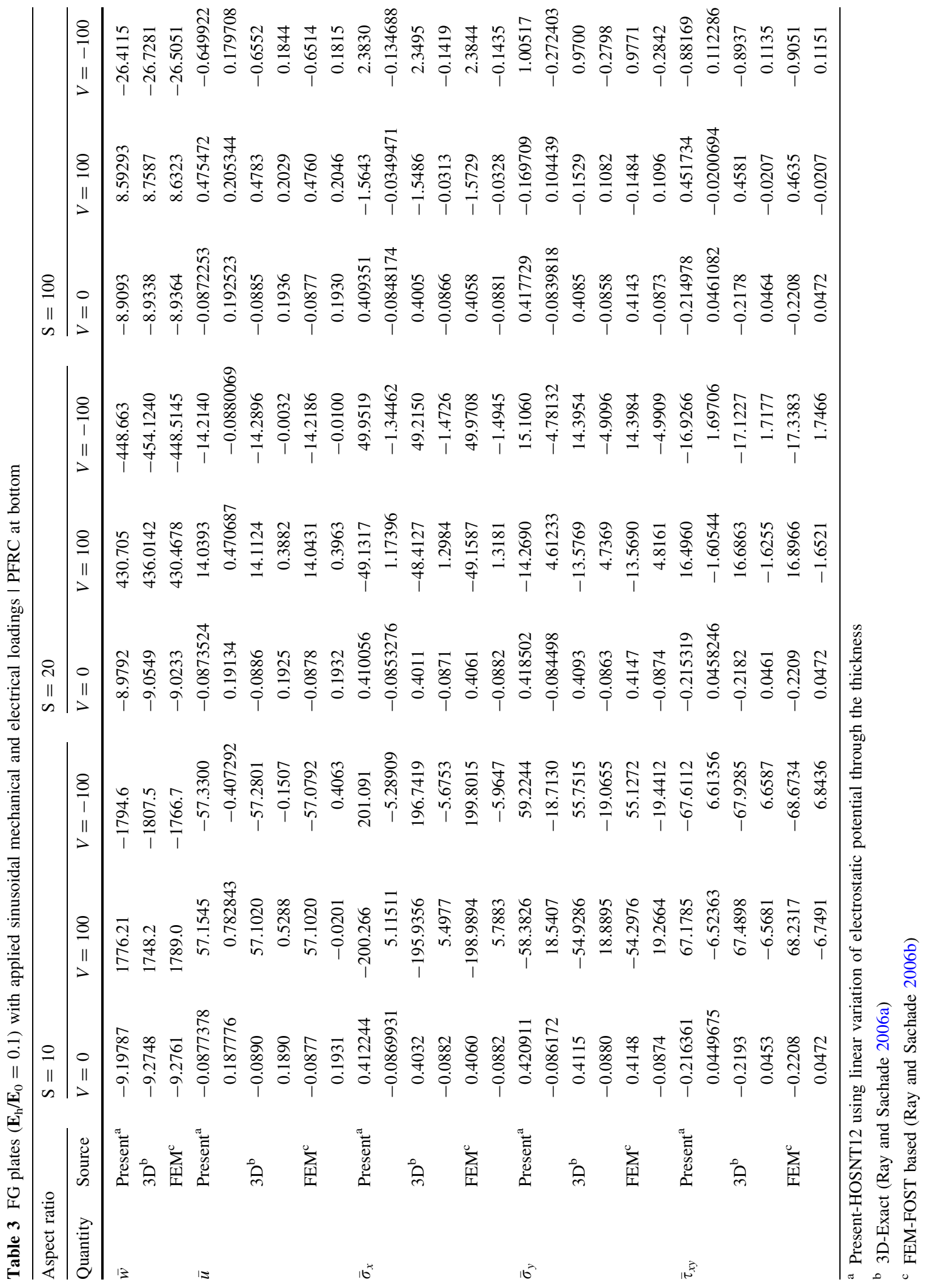




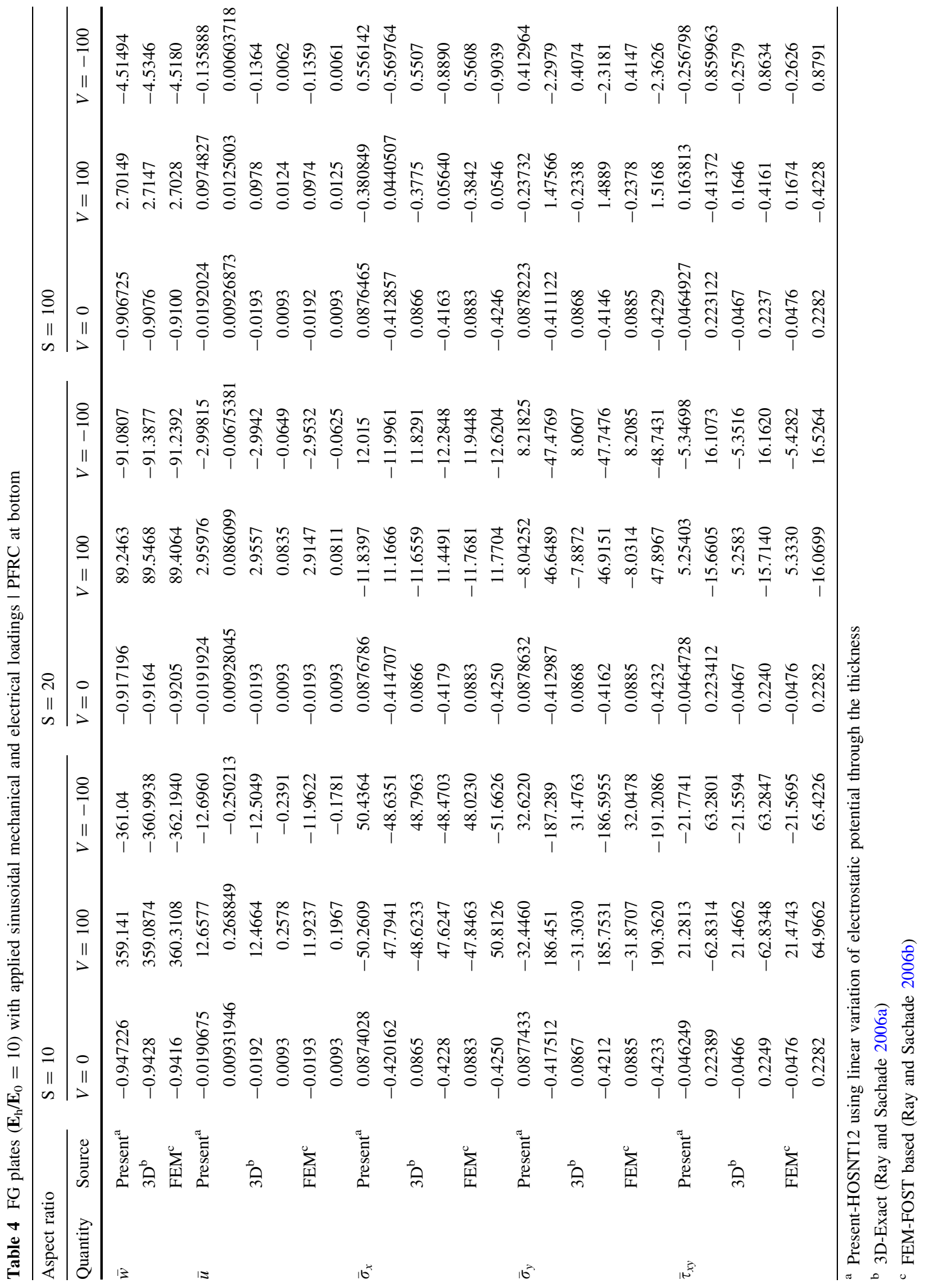


displacement, in-plane normal stresses and transverse shear stress are evaluated and their comparison with exact and FEM solutions are presented in Tables and Figures.

Tables 1, 2, 3, and 4 show comparison of numerical results of for the cases I, II, III and IV, respectively.

Figure $2 \mathrm{a}$ and $\mathrm{b}$ demonstrates normalized variation of transverse displacement $(\bar{w})$ and in-plane displacement $(\bar{u})$ through the thickness of thin FG plate $(S=100)$, respectively. Transverse displacement is constant through the thickness of the FG layer while, in-plane displacement is linear. Normalized variations of inplane normal stresses $\left(\bar{\sigma}_{x}, \bar{\sigma}_{y}\right)$ through the thickness of thin FG plate are exhibited in Fig. 3a and b. Variation of in-plane normal stress $\left(\bar{\sigma}_{x}\right)$ at top of FG plate is more than in-plane normal stress $\left(\bar{\sigma}_{y}\right)$. This is due to effectiveness of piezoelectric stress coefficient only
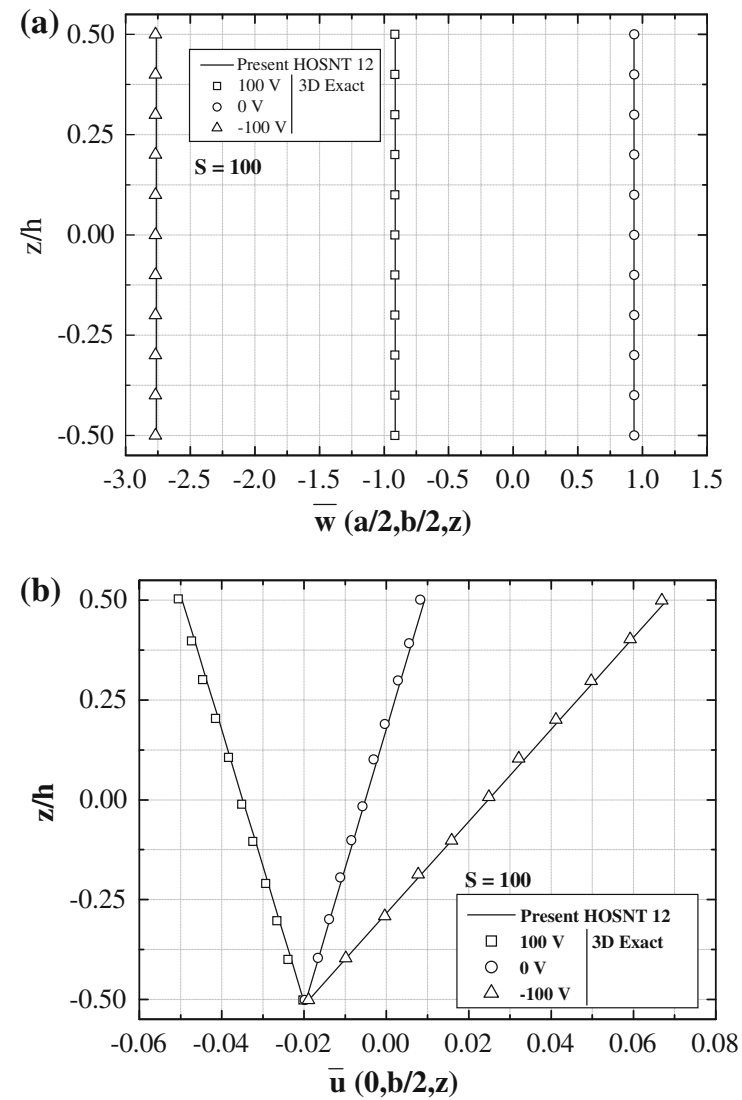

Fig. 2 Variation of normalized (a) Transverse displacement $\bar{w}$ (b) In-plane displacement $\bar{u}$ through the thickness $(z / h)$ of a Functionally Graded (FG) plate $\left(E_{h} / E_{0}=10\right)$ simply supported on all edges with or without applied voltage to the piezoelectric layer
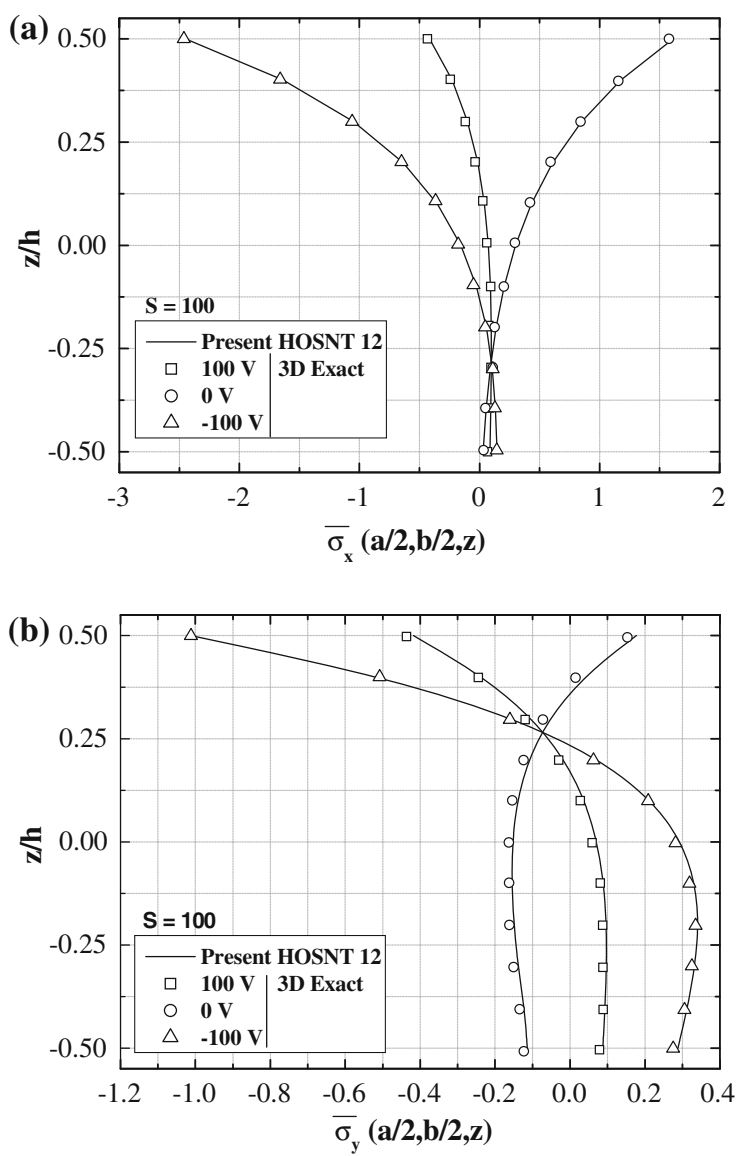

Fig. 3 Variation of normalized (a) In-plane normal stress $\bar{\sigma}_{x}$ (b) In-plane normal stress $\bar{\sigma}_{y}$ through the thickness $(z / h)$ of a Functionally Graded (FG) plate $\left(E_{h} / E_{0}=10\right)$ simply supported on all edges with or without applied voltage to the piezoelectric layer

along $x$-axis. Present HOSNT12 results are closed to exact solution for with or without applied voltages. Figure $4 \mathrm{a}$ and $\mathrm{b}$ demonstrates normalized variation of in-plane shear stress $\left(\bar{\tau}_{x y}\right)$ and transverse normal stress $\left(\bar{\sigma}_{z}\right)$ through the thickness of thin FG plate. Results of transverse shear stress $\left(\bar{\tau}_{y z}, \bar{\tau}_{x z}\right)$ closely agree with exact results for both the voltages as shown in Fig. 5a and b, respectively. Traction free conditions for transverse shear stress $\left(\bar{\tau}_{y z}\right)$ can be observed at bottom as well as at top of FG plate, but maximum shear traction is seen at top of FG plate where actuator is placed.

\section{Conclusions}

In this paper a complete analytical solution for statics of FG plate attached with distributed PFRC actuator 

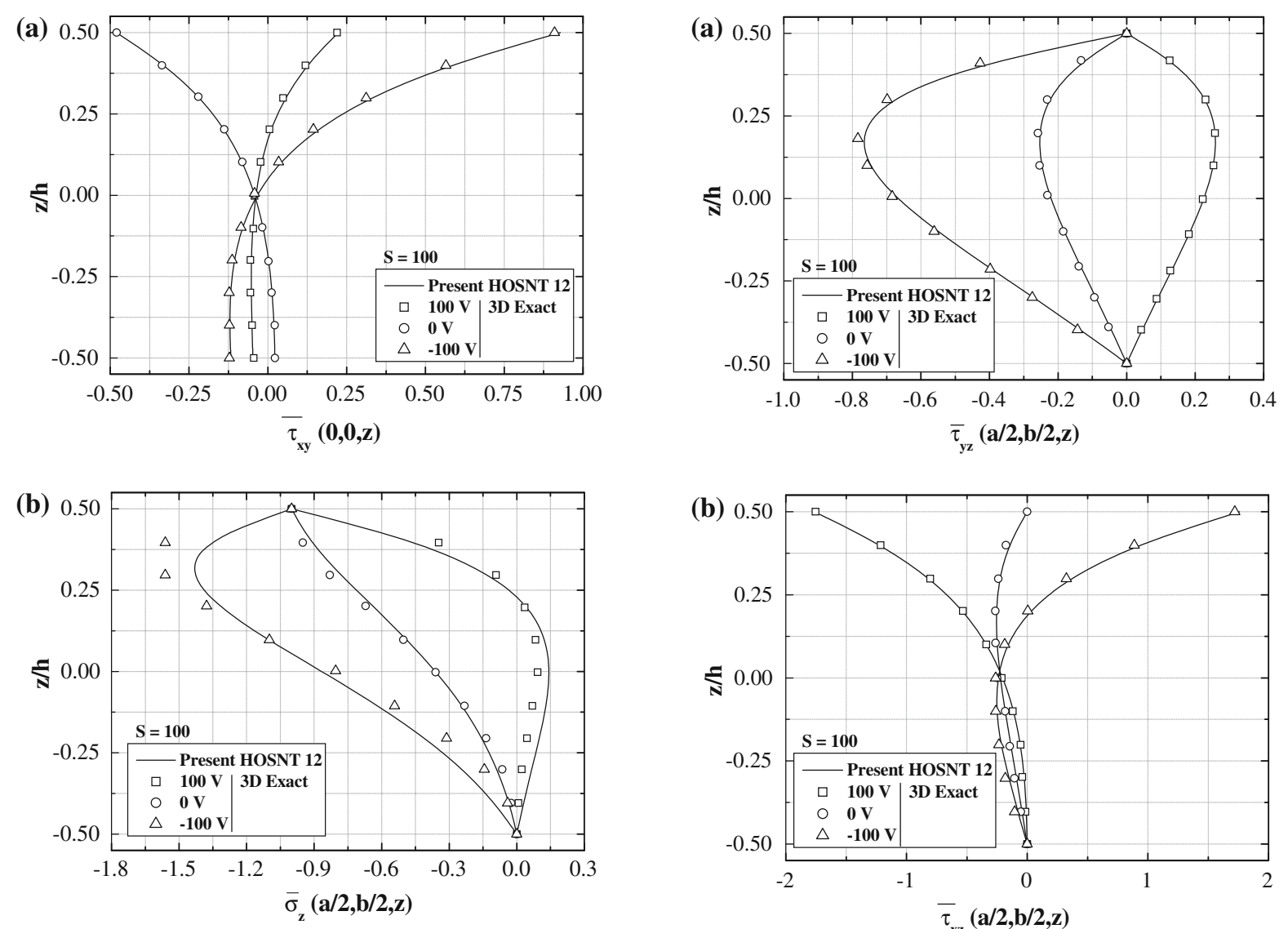

Fig. 4 Variation of normalized (a) In-plane shear stress $\bar{\tau}_{x y}$ (b) Transverse normal stress $\bar{\sigma}_{z}$ through the thickness $(z / h)$ of a Functionally Graded (FG) plate $\left(E_{h} / E_{0}=10\right)$ simply supported on all edges with or without applied voltage to the piezoelectric layer

under electromechanical load is presented. A higher order shear and normal deformation theory is used to model the elastic responses of FG plate subjected to voltages. Linear layer wise approximation of the electrostatic potential is proposed in the present model. Comparative numerical results and across the thickness variations of displacements and stresses are presented. Linear and constant variations of in-plane and transverse displacements are observed. Considerable effect of actuation at the interface is observed in case of in-plane normal stress and transverse shear stress along $x$-axis as compared to their effects along $y$ axis. Present model performs excellently to predict the variations in these cases.

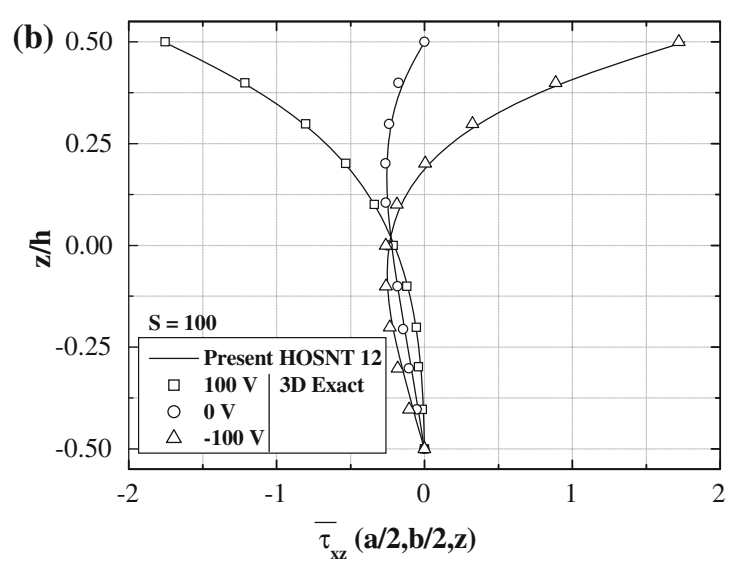

Fig. 5 Variation of normalized (a) Transverse shear stress $\bar{\tau}_{y z}$ (b) Transverse shear stress $\bar{\tau}_{x z}$ through the thickness $(z / h)$ of a Functionally Graded (FG) plate $\left(E_{h} / E_{0}=10\right)$ simply supported on all edges with or without applied voltage to the piezoelectric layer

\section{References}

Batra, R.C., Vel, S.S.: Exact solution for thermoelastic deformations of functionally graded thick rectangular plates. AIAA J. 40(7), 1421-1433 (2001)

Heyliger, P.: Static behavior of laminated elastic/piezoelectric plates. AIAA J. 32, 2481-2484 (1994)

Heyliger, P.: Exact Solutions for simply supported laminated piezoelectric plates. ASME J. Appl. Mech. 64, 299-306 (1997)

Kant, T.: Numerical analysis of thick plates. Comput. Methods Appl. Mech. Eng. 31, 1-18 (1982)

Kant, T., Manjunatha, B.S.: An unsymmetric FRC laminate $C^{\circ}$ finite element model with 12 degrees of freedom per node. Eng. Comput. 5(4), 300-308 (1988)

Kant, T., Manjunatha, B.S.: On accurate estimation of transverse stresses in multilayer laminates. Comput. Struct. 50, 351-365 (1994) 
Kant, T., Owen, D., Zinkiewicz, O.C.: A refined higher-order $\mathrm{C}^{\mathrm{O}}$ plate bending element. Comput. Struct. 15, 177-183 (1982)

Kant, T., Swaminathan, K.: Analytical solutions for the static analysis of laminated composite and sandwich plates based on a higher order refined theory. Compos. Struct. 56, 329-344 (2002)

Kapuria, S., Dumir, P.C.: Coupled FSDT for piezothermoelectric hybrid rectangular plate. Int. J. Solids Struct. 37, 6131-6153 (2000)

Mallik, N., Ray, M.C.: Effective coefficients of piezoelectric fiber reinforced composites. AIAA J. 41(4), 704-710 (2003)

Pagano, N.J.: Exact solution for rectangular bidirectional composites and sandwich plates. J. Compos. Mater. 4, 20-34 (1970)

Pandya, B.N., Kant, T.: A consistent refined theory for flexure of a symmetric laminate. Mech. Res. Commun. 14, 107-113 (1987)

Pandya, B.N., Kant, T.: A refined higher-order generally orthotropic $\mathrm{C}^{0}$ plate bending element. Comput. Struct. 28, 119-133 (1988)

Ray, M.C., Sachade, H.M.: Exact Solutions for the Functionally Graded Plates Integrated With a Layer of Piezoelectric Fiber-Reinforced Composite. ASME J. Appl. Mech. 73(4), 622-633 (2006a)
Ray, M.C., Sachade, H.M.: Finite element analysis of smart functionally graded plates. Int. J. Solids Struct. 43, 54685484 (2006b)

Ray, M.C., Bhattacharya, R., Samanta, B.: Exact Solutions for static analysis of intelligent structures. AIAA J. 31, 16841691 (1993)

Reddy, J.N.: A simple higher order theory for laminated plates. ASME J. Appl. Mech. 51, 745-752 (1984)

Reddy, J.N., Cheng, Z.Q.: Three-dimensional solutions of smart functionally graded plates. ASME J. Appl. Mech. 68(3), 234-241 (2001)

Sankar, B.V.: An elasticity solution for functionally graded beams. Compos. Sci. Tech. 61(5), 689-696 (2001)

Smith, W.A., Auld, B.A.: Modeling 1-3 composite piezoelectrics: thickness mode oscillations. IEEE Trans. Ultrason. Ferroelectr. Frequency Control 31(1), 40-47 (1991)

Tiersten, H.F.: Linear Piezoelectric Plate Vibrations. Plenum Press, New York (1969)

Tiersten, H.F., Mindlin, R.D.: Forced vibrations of piezoelectric crystal plates. Quart. Appl. Math. 20, 107-119 (1962)

Vel, S.S., Batra, R.C.: Three-dimensional analytical solution for hybrid multilayered piezoelectric plates. ASME J. Appl. Mech. 67, 558-567 (2000) 